\title{
Competencias para la Empleabilidad de los Futuros Maestros de Educación Primaria: Una Mirada a su Proceso de Inserción Socio-laboral
}

\author{
Employability Competences of Future Primary Education Teachers: A look at \\ their Social and Labor Insertion Process \\ Competências para a Empregabilidade dos Futuros Mestres do Ensino Básico: \\ Um olhar para seu Processo de Inserção Social e no Trabalho
}

Cristina González Lorente* https://orcid.org/0000-0001-7211-3862

Departamento de Métodos de Investigación y Diagnóstico en Educación, Facultad de Educación, Universidad de Murcia, Murcia, España.

Nuria Rebollo-Quintela** https://orcid.org/0000-0001-9026-0794

Departamento de Didácticas Específicas y Métodos de Investigación y Diagnóstico en Educación, Facultad de Ciencias de la Educación, Universidade da Coruña, A Coruña, España.

$\nabla$

Recibido: 05-04-18 Revisado: 13-05-18 Aceptado: 01-10-18 Publicado: 20-12-18

- Resumen. En la sociedad del aprendizaje, uno de los retos para los maestros en formación es la necesidad de definir y desarrollar un conjunto de competencias profesionales en su formación inicial, esenciales en su entrada al mercado laboral. En este trabajo, se tuvo como objetivo ahondar en las competencias para la empleabilidad que desarrollan los estudiantes del Grado en Educación Primaria, la relevancia que le confieren para su proceso de inserción sociolaboral así como su percepción global para afrontarlo. Se realizó un estudio inter-universitario con un total de 210 estudiantes de último curso en la Universidad de Murcia y la Universidade da Coruña, ambas españolas. La recogida de información se realizó a partir del Cuestionario de Orientación e Inserción Laboral, validado previamente. Los resultados mostraron escasas diferencias entre las valoraciones de los estudiantes de ambas universidades, que coinciden en señalar una mayor relevancia de las competencias frente al nivel de desarrollo en aula. En
Palabras clave: competencia; educación primaria; maestro; inserción sociolaboral. 
definitiva, la atención se debe dirigir a aunar esfuerzos en el estado español en la formación inicial de los maestros de educación primaria, para que puedan adquirir un conjunto de competencias que les facilite, además de su incursión laboral, su continua mejora y actualización en el ejercicio de su desarrollo profesional, social y personal.

- Abstract. In the learning society, one of the challenges for teachers in training is the need to define and develop a set of professional skills in their initial training, essential for their subsequent entry into the labor market. In this work, the aim was to delve into the employability skills developed by students in the Primary Education undergraduate study program, their relevance for their process of socio-labor insertion, as well as their global perception to face it. An inter-university study was carried out with a total of 210 students in their final year at Universidad de Murcia and Universidade da Coruña, both Spanish. The collection of information was carried out on the basis of the previously validated Labor Counseling and Insertion Questionnaire. The results showed few differences between the assessments of the students of both universities, which coincide in pointing out to a greater relevance of the competences as opposed to the level of development in the classroom. In short, attention should be directed to joining efforts by the Spanish government in the initial training of primary education teachers, so that they can acquire a set of competences that will allow a continuous improvement and updating in their professional, social and personal performance, in addition to their career inception.

- Resumo. Na sociedade do aprendizado, um dos desafios para os professores em formação é a necessidade de definir e desenvolver um conjunto de competências profissionais em sua formação inicial, essenciais na sua inserção posterior no mercado de trabalho. Neste estudo, teve-se como objetivo aprofundar nas competências para a empregabilidade desenvolvidas por estudantes do curso de Ensino Básico, a relevância conferida para seu processo de inserção social e no trabalho, assim como sua percepção global para enfrentá-lo. Realizouse um estudo entre universidades com um total de 210 estudantes do último curso na Universidade de Múrcia e a Universidade da Corunha, ambas espanholas. O levantamento de informação foi realizado a partir do Questionário de Orientação e Inserção no Trabalho, validado previamente. Os resultados mostraram poucas diferenças entre as valorações dos estudantes de ambas as universidades, que coincidem em apontar uma maior relevância das competências diante do nível de desenvolvimento na sala de aula. Em definitiva, o foco devese dirigir a unir esforços no estado espanhol para a formação inicial de professores de ensino básico, para que possam adquirir um conjunto de competências que lhes facilitem, além de sua incursão no mundo do trabalho, sua melhora contínua e atualização no exercício de seu desenvolvimento profissional, social e pessoal.
Keywords: competence, primary education; teacher; social and career insertion

Palavras chave: competências; ensino básico; professor; inserção social e no trabalho. 


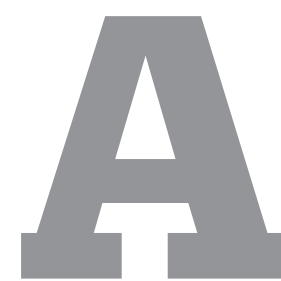

1 pensar en las posibilidades de inserción laboral de un maestro de educación primaria, inmediatamente la palabra "oposición" viene a la mente de gran parte de los futuros graduados en estos estudios. Es cierto que se trata de una profesión regulada, con una gran oferta de empleo en el sector público entre la salida al mercado laboral más significativa, pero actualmente se constata que los efectos de la fuerte crisis socio-económica acaecida durante la última década, atenúa un nuevo escenario de incertidumbre e inestabilidad en el proceso de inserción socio-laboral que afecta de forma incipiente a la población joven, incluidos aquellos que cuentan con una titulación universitaria de carácter profesionalizador, como es el caso de los maestros.

La transición a la vida activa ocupa períodos de tiempo cada vez más alargados y representa uno de los momentos más críticos en el desarrollo de la carrera de cualquier universitario (Helyer \& Lee, 2014; Pineda, Agud, \& Ciraso, 2016; Stiwne \& Alves, 2010). Lejos queda, como señala Contreras (2014), el patrón tradicional de generaciones anteriores en las que, de forma breve y directa, se pasaba de "una situación de partida con dedicación completa al estudio dentro del sistema educativo, a una posición de llegada a un empleo estable a tiempo completo" ( $p$. 74). Este cambio de escenario está repleto de obstáculos para dicha transición en los jóvenes, incluidos aquellos que han dedicado gran parte de su tiempo y esfuerzo a cursar estudios de educación superior y adquiere especial relevancia con la introducción de las universidades españolas en el Espacio Europeo de Educación Superior (EEES). Desde este momento, resulta fundamental promover el desarrollo de competencias para que los futuros egresados puedan adaptarse de forma continua a un entorno social y económico en constante desequilibrio, al mismo tiempo que se incrementan las investigaciones para conocer mejor las percepciones y creencias que poseen los universitarios hacia el empleo y comprender así, su influencia en la forma de abordar la transición a la vida activa.

En el caso de los estudiantes de educación primaria, este complejo proceso hacia la inserción socio-laboral viene acompañado de la aparición de nuevas estructuras curriculares, de continuas actualizaciones en las metodologías de enseñanza centradas en el alumnado como protagonista o de nuevos proyectos educativos que trabajan directamente la interdisciplinariedad del conocimiento, sin olvidarnos de la "ampliación de la mirada del docente hacia lo social para cubrir facetas desatendidas por la sociedad y por las familias" (Prats, 2016, p. 22). Todos estos y demás fenómenos, provocan un parangón sin precedentes en la tradicional historia de la formación de los maestros que, como señalan Hudson, Zgaga y Astrand (2010), demandan nuevas y cambiantes exigencias pedagógico-didácticas, tanto dentro como fuera del aula.

Estos grandes retos y demandas que deben asumir los nuevos maestros, justifican la necesidad de definir y desarrollar un conjunto de competencias profesionales esenciales para su formación inicial y posterior entrada al mercado laboral. Unas competencias que, más allá 
del dominio técnico y metodológico del proceso de enseñanza-aprendizaje, promuevan la inserción, continuidad y perfeccionamiento profesional a lo largo de la carrera docente. Rosales (2013) indica que sería utópico pensar en el desarrollo absoluto de todas y cada una de las competencias incluidas en los planes deestudios universitarios tras finalizar la formación inicial. De hecho, en un marco educativo como el actual, basado en la adquisición de competencias, existen todavía hoy muchos docentes que, paradójicamente, no se sienten competentes para emprender la labor profesional como maestros y maestras una vez finalizado el período de formación inicial (Herraiz, 2015).

A lo largo de los últimos años, diferentes estudios de investigación dirigen sus esfuerzos hacia el análisis de las competencias que debería poseer realmente un maestro, entre otros, Mendoza y Covarrubias (2014), Grover (2015), Rodríguez-Gómez, Armengol y Meneses, (2017) o Rosales (2013) siendo muchos y diversos los intentos para responder a las preguntas sobre qué se debería aprender y qué competencias se deberían desarrollar en esta titulación universitaria, pero como señala Medina y Pérez Cabrera (2017), en escasas ocasiones, se cuenta con la opinión y experiencia de los principales implicados para contestarlas, esto es, los futuros egresados.

Por esta razón, este trabajo pretende ahondar un poco más en las competencias para la empleabilidad que desarrollan los estudiantes del Grado en Educación Primaria, desde sus propias creencias y percepciones, a partir de la clasificación de competencias realizada por Martínez Clares y González Lorente (2018); una propuesta que congrega, de forma sistematizada y contrastada, un conjunto de competencias de acción profesional que pueden considerarse comunes y transversales a todo profesional del siglo XXI. Dicha clasificación, enfatiza el carácter aplicativo y contextualizado de las competencias en el actual mercado laboral, con independencia de la disciplina académica. Se trata, por tanto, de un horizonte más que de una experiencia o modelo consolidado, cuyo objetivo es, a través de un conjunto de competencias, orientar la transición a la vida activa y promover la empleabilidad de los universitarios en un contexto socio-laboral con constantes variaciones.

Desde este enfoque general sobre competencias para la empleabilidad aplicables a la formación universitaria en cualquier titulación o rama del conocimiento, se realiza un estudio interuniversitario que permite profundizar en este tópico, tanto en el desarrollo de las competencias durante la carrera, como en la relevancia de las mismas para el proceso de inserción socio-laboral, mostrando finalmente una visión global para afrontar dicho proceso desde la propia percepción de los estudiantes de último curso del Grado en Educación Primaria de dos universidades españolas, como son, la Universidad de Murcia (UM) y la Universidade da Coruña (UDC). Todo este planteamiento se concreta en un triple objetivo:

1. Conocer y analizar el desarrollo y relevancia que los estudiantes de último curso de Grado en Educación Primaria de las dos universidades analizadas (UM y UDC) le 
confieren a un conjunto de competencias para su proceso de inserción socio-laboral.

2. Identificar y contrastar posibles diferencias significativas en el desarrollo y relevancia de estas competencias en función de la universidad en la que se realiza el Grado en Educación Primaria.

3. Considerar y evaluar la percepción que manifiesta el alumnado respecto a su preparación para enfrentarse al proceso de inserción socio-laboral en ambas universidades.

\section{MÉTODO}

\section{Diseño}

Para la realización de este trabajo se parte de un enfoque metodológico cuantitativo, al estudiar el desarrollo y relevancia de un conjunto de competencias como variables cuantificadas. Dentro de este enfoque, se recurre a un diseño de investigación no experimental, en el que los participantes pertenecen al Grado en Educación Primaria como grupo determinado, sin manipulación intencional de las variables niasignación al azar (Hernández, Fernández, \& Baptista, 2007). De forma más específica, dicho diseño es, asimismo, transversal y descriptivo, de acuerdo con el momento concreto de recogida de información y el carácter de los objetivos propuestos.

\section{Participantes}

En este trabajo participan un total de 210 estudiantes de último curso de Grado distribuidos entre la Universidad de Murcia (UM) y de la Universidade da Coruña (UDC) que cursan el Grado en Educación Primaria en las Facultades de Ciencias de la Educación de sendas Instituciones de Educación Superior. La elección de la muestra objeto de estudio radica en dos aspectos fundamentales: por un lado, son quienes tienen más próximo su proceso de inserción sociolaboral y por el otro, por el bagaje formativo acumulado durante cuatro años de experiencia que les otorga una mejor perspectiva para valorar el desarrollo de competencias en su titulación.

Respecto de las características de la muestra, la distribución es similar tanto en número como en feminización de la misma (ver Tabla 1) ya que, en ambos casos, más del 70\% del alumnado encuestado es femenino. Por su parte, la edad de los participantes se encuentra en el intervalo entre los 20 y 47 años, con una media de 22.96 años ( $D E=3.80$ ) y con mayor frecuencia entre los 20-25 años (82.9\%). Si profundizamos en su descripción nos encontramos con que es un alumnado con buen expediente académico, ya que el 93.4\% en el caso de la UDC y del 81.7\% en la UM posee una nota media de notable. Por último, si atendemos a su situación laboral en el momento encuestado, el 38.7\% del alumnado de la UDC y el 50\% de la UM tenía como ocupación a tiempo completo los estudios, y el resto de la muestra encuestada se encontraba compaginándolos con trabajos. 
Tabla 1

Distribución de la muestra por universidad participante y sexo

\begin{tabular}{|lccccccc|}
\hline & Hombres (n) & $\%$ & Mujeres (n) & $\%$ & Otro (n) & \% & Total \\
\hline Universidad de Murcia (UM) & 29 & 27.9 & 75 & 72.1 & 0 & 0 & 104 \\
\hline Universidade da Coruña (UDC) & 22 & 20.75 & 82 & 77.35 & 2 & 1.9 & 106 \\
\hline
\end{tabular}

\section{Instrumento}

Para la recogida de información, se utiliza el Cuestionario de Orientación e Inserción Laboral (COIL) de Martínez Clares y González Lorente (2018) con un índice de fiabilidad o consistencia interna alto (coeficiente de Cronbach $\alpha=.898$ ). La finalidad de dicho instrumento es recoger las impresiones y valoraciones de los estudiantes de último curso de Grado en relación con el proceso de inserción socio-laboral, a partir de los tres grandes bloques de contenido en los que se divide el mismo (ver Tabla 2).

\section{Tabla 2}

Estructura del Cuestionario de Orientación e Inserción Laboral

\begin{tabular}{|c|l|}
\hline $\begin{array}{c}\mathbf{1}^{\circ} \text { Bloque: Información } \\
\text { general }\end{array}$ & $\begin{array}{l}\text { Siete preguntas (abiertas y cerradas) para conocer datos personales } \\
\text { genéricos (edad y sexo), así como su situación formativa y laboral actual }\end{array}$ \\
\hline \multirow{2}{*}{$\begin{array}{c}\mathbf{2}^{\circ} \text { Bloque: Formación } \\
\text { universitaria }\end{array}$} & $\begin{array}{l}\text { Una escala de competencias para la empleabilidad tipo Likert con cinco } \\
\text { niveles de estimación (1=Nada; 5=Mucho), donde se valora tanto el } \\
\text { Desarrollo como la Relevancia. }\end{array}$ \\
\cline { 2 - 2 } & $\begin{array}{l}\text { Una escala de satisfacción formación universitaria para su proceso de } \\
\text { inserción socio-laboral) tipo Likert (1=Nada; } 5=M u c h o) .\end{array}$ \\
\hline $\begin{array}{c}3^{\circ} \text { Bloque: Intereses y } \\
\text { búsqueda de empleo }\end{array}$ & $\begin{array}{l}\text { Cinco preguntas semicerradas sobre los intereses, expectativas y recursos } \\
\text { relacionados con el proceso de inserción laboral y la búsqueda de empleo. }\end{array}$ \\
\hline
\end{tabular}

A lo largo de los diferentes bloques, se incluyen preguntas de valoración cualitativa y cuantitativa como parte del enfoque metodológico mixto de una investigación más amplia de la que forma parte este trabajo. En particular, se centra únicamente en el segundo bloque sobre la experiencia de los estudiantes en relación con su formación universitaria y preparación para su inserción socio-laboral. Dentro de este bloque aparece la escala de competencias donde se incorporan un total de 19 elementos basados en la clasificación aportada por Martínez Clares y González Lorente (2018) a partir de una doble dimensión de análisis que engloba tanto el desarrollo como la relevancia de dichas competencias. Cabe mencionar en este sentido, que la 
dimensión de Desarrollo permite realizar una aproximación al nivel de trabajo e incidencia sobre estas competencias durante la formación de Grado, mientras que la dimensión de Relevancia, se entiende como la importancia que el alumnado le confiere a cada una de ellas para su futura inserción en el mercado laboral.

\section{Procedimiento}

El proceso de investigación que se aborda en este trabajo viene delimitado por una serie de fases secuenciales y sistemáticas que se inician con el problema de investigación. Dicho problema se enmarca en la situación de desconcierto e incertidumbre que experimentan actualmente los egresados universitarios a la hora de enfrentarse a su proceso de inserción socio-laboral. Para conocer mejor esta situación y plantear programas de orientación profesional que den respuesta a tales momentos de cambio, se inicia este estudio con la búsqueda de información en profundidad que deriva en la delimitación de los objetivos por alcanzar. A continuación, se diseña el Cuestionario de Orientación e Inserción Laboral (COIL) para la recogida de información. Durante el curso académico 2016/2017 se realiza una prueba piloto del cuestionario, que también se somete a un completo proceso de validación de contenido (a través de la técnica de juicio de expertos) y constructo (mediante la elaboración de un modelo de ecuaciones estructurales) para garantizar su validez. Tras el diseño y validación del cuestionario COIL, se contacta con el profesorado con docencia en el último curso en la UM y en la UDC, para acordar el horario de clase más adecuado para la recogida de información durante el curso 2017/2018. A los estudiantes que desean participar en ambas universidades, se les recuerda en todo momento el carácter voluntario, anónimo y confidencial de los datos recabados, que posteriormente se analizan con la ayuda del programa estadístico SPSS v23. Se recurre a la estadística descriptiva (la media aritmética y desviación típica) y a la estadística inferencial para detectar las diferencias significativas entre las dimensiones estudiadas, así como entre los estudiantes de ambas universidades, asumiendo en los cálculos inferenciales un nivel de significación de $p \leq .005$. Por último, se realiza la interpretación y discusión de los resultados para presentar este trabajo de investigación.

\section{Resultados}

Para una mayor comprensión y profundización, se van a presentar los resultados con relación a cada uno de los objetivos propuestos inicialmente.

Para dar respuesta al primer objetivo: conocer y analizar el desarrollo y relevancia que los estudiantes de último curso de Grado en Educación Primaria de las dos universidades analizadas (UM y UDC) le confieren a un conjunto de competencias profesionales en su proceso de inserción socio-laboral, se estudian por separado las dos instituciones de educación superior.

En primer lugar, se analiza el grado en el que se han desarrollado en el título impartido en la UM, las competencias para la inserción laboral que componen el instrumento diseñado, 
con base en la perspectiva del discente. Así mismo, se determina en qué medida estos las consideran de importancia para su inserción en el mercado laboral. Se constata que, tanto en el caso de la relevancia otorgada como en el nivel de desarrollo de las competencias en el aula, en la Universidad de Murcia existe un alto grado de homogeneidad en las respuestas dadas por el alumnado, ya que la desviación típica en todos los ítems analizados es cercana a 1.

Los resultados muestran un gran nivel de desarrollo de todas las competencias, con medias iguales o superiores a 3.25, siendo "el trabajo en equipo y cooperación" ( $\bar{X}=4.36)$ la que consideran haber abordado en mayor medida, seguida de la "responsabilidad y perseverancia" $(\bar{X}=3.78)$, "la toma de decisiones" $(\bar{X}=3.75)$, "la conciencia de uno mismo" $(\bar{X}=3.71)$ y "las habilidades de comunicación" ( $\bar{X}=3.71)$. Sin embargo, las competencias que perciben como menos desarrolladas a los largo de sus estudios, son "el liderazgo" ( $\bar{X}=3.25)$ y "el compromiso con la organización" ( $\bar{X}=3.25)$ y la "búsqueda por la excelencia" ( $\bar{X}=3.28)$.

El análisis del nivel de relevancia otorgada a estas competencias laborales arroja medias superiores a 4. Dentro de esta, destaca la importancia del "trabajo en equipo y cooperación" $(\bar{X}=4.56)$, "la capacidad de aprendizaje y adaptación" $(\bar{X}=4.57)$, las “habilidades de comunicación” $(\bar{X}=4.62)$ y la "responsabilidad y perseverancia" $(\bar{X}=4.62)$, mientras que "el liderazgo" $(\bar{X}=4.05)$ y el "compromiso por la organización" $(\bar{X}=4.12)$ son las competencias con menor valoración en relación con la importancia para su inclusión laboral. No obstante, como se observa en la Tabla 3 en la totalidad de las competencias estudiadas, la media obtenida en el caso de la relevancia es siempre superior al nivel de desarrollo en el aula del Grado en Educación Primaria de la Universidad de Murcia.

Al aplicar la prueba de Wilcoxon, prueba no paramétrica para dos muestras relacionadas, se aprecia que esta diferencia observada entre el nivel de desarrollo y relevancia, es estadísticamente significativa ( $p \leq .05)$, en todas las competencias analizadas (ver Tabla 3 ), en el caso de la Universidad de Murcia.

\section{Tabla 3}

Desarrollo y relevancia de las competencias de la Universidad de Murcia

\begin{tabular}{|lccccc|}
\hline \multirow{2}{*}{ Competencias profesionales } & \multicolumn{2}{c}{ Desarrollo } & \multicolumn{2}{c|}{ Relevancia } & Sig (p) \\
\cline { 2 - 5 } & Media & Desv. típ. & Media & Desv. típ. & Wilcoxon \\
\hline Conciencia de uno mismo (C1) & 3.71 & 0.966 & 4.44 & 0.774 & $<.001$ \\
\hline Análisis, síntesis y crítica (C2) & 3.37 & 0.904 & 4.13 & 0.789 & $<.001$ \\
\hline Organización y planificación (C3) & 3.62 & 0.894 & 4.46 & 0.637 & $<.001$ \\
\hline Habilidades de comunicación (C4) & 3.71 & 0.921 & 4.62 & 0.671 & $<.001$ \\
\hline Responsabilidad y perseverancia (C5) & 3.78 & 0.965 & 4.62 & 0.612 & $<.001$ \\
\hline Toma de decisiones (C6) & 3.75 & 0.953 & 4.46 & 0.667 & $<.001$ \\
\hline Orientación a las personas (C7) & 3.34 & 1.171 & 4.32 & 0.804 & $<.001$ \\
\hline Trabajo en equipo y cooperación (C8) & 4.36 & 0.835 & 4.56 & 0.708 & .047 \\
\hline
\end{tabular}




\begin{tabular}{|lccccc|}
\hline \multirow{2}{*}{ Competencias profesionales } & \multicolumn{2}{c}{ Desarrollo } & \multicolumn{2}{c}{ Relevancia } & Sig (p) \\
\cline { 2 - 5 } & Media & Desv. típ. & Media & Desv. típ. & Wilcoxon \\
\hline Capacidad de aprendizaje y adaptación (C9) & 3.56 & 1.050 & 4.57 & 0.707 & $<.001$ \\
\hline Flexibilidad y orientación al cambio (C10) & 3.42 & 0.921 & 4.30 & 0.811 & $<.001$ \\
\hline Motivación por el logro (C11) & 3.42 & 1.146 & 4.49 & 0.724 & $<.001$ \\
\hline Compromiso con la organización (C12) & 3.25 & 1.022 & 4.12 & 0.896 & $<.001$ \\
\hline Capacidad para trabajar bajo presión (C13) & 3.67 & 1.242 & 4.18 & 0.932 & .002 \\
\hline $\begin{array}{l}\text { Resolución de conflictos y técnicas de } \\
\text { negociación (C14) }\end{array}$ & 3.31 & 1.115 & 4.47 & 0.763 & $<.001$ \\
\hline Búsqueda de la excelencia (C15) & 3.28 & 1.144 & 4.25 & 0.797 & $<.001$ \\
\hline Innovación (C16) & 3.37 & 1.116 & 4.52 & 0.623 & $<.001$ \\
\hline Emprendimiento (C17) & 3.31 & 1.124 & 4.48 & 0.724 & $<.001$ \\
\hline Liderazgo (C18) & 3.25 & 1.121 & 4.05 & 0.959 & $<.001$ \\
\hline $\begin{array}{l}\text { Capacidad de resiliencia y tolerancia a la } \\
\text { frustración (C19) }\end{array}$ & 3.32 & 1.151 & 4.34 & 0.832 & $<.001$ \\
\hline
\end{tabular}

En un análisis pormenorizado de la Universidade da Coruña se evidencia, al igual que en el caso de la Universidad de Murcia, que existe un alto grado de homogeneidad en las respuestas dadas por el alumnado, siendo esta superior en las valoraciones relacionadas con la relevancia.

Las competencias profesionales se han desarrollado en su totalidad a lo largo del título, el alumnado otorga una mayor puntuación al "trabajo en equipo" ( $\bar{X}=3.99)$, seguido de "la responsabilidad y la perseverancia" ( $\bar{X}=3.79)$ y las "habilidades de comunicación" ( $\bar{X}=3.75)$. Por el contrario, considera que se han trabajado en menor medida el "emprendimiento" $(\bar{X}=3.15)$ y la "capacidad de resiliencia y tolerancia a la frustración" ( $\bar{X}=3.18)$.

Cuando se examina la importancia concedida a estas competencias para la inserción laboral, se aprecia que a excepción de 1, el resto obtiene medias superiores a 4, siendo las que obtienen mayor reconocimiento por parte del alumnado "las habilidades de comunicación" ( $\bar{X}$ =4.55) y la "capacidad de aprendizaje y adaptación" ( $\bar{X}=4.49)$ sin embargo son el "liderazgo" ( $\bar{X}=3.89)$, el "compromiso con la organización" $(\bar{X}=4.02)$, "la capacidad para trabajar bajo presión” $(\bar{X}=4.06)$ y el "emprendimiento" ( $\bar{X}=4.08)$ las competencia menos valorada.

Existen diferencias en el alumnado de la Universidade da Coruña en cuanto a los dos aspectos objeto de estudio, es decir, entre la relevancia otorgada a las competencias profesionales y el grado de desarrollo de las mismas a lo largo de la carrera, siendo siempre superior la importancia conferida. Para determinar, en qué medida estas diferencias señaladas son estadísticamente significativas, se aplica la prueba no paramétrica de Wilcoxon. Los resultados obtenidos evidencian diferencias estadísticamente significativas ( $p \leq .05)$, para el conjunto de las competencias siendo la relevancia, como en el caso de la Universidad de Murcia, superior al desarrollo (ver Tabla 4). 
Tabla 4

Desarrollo y relevancia de las competencias de la Universidade da Coruña

\begin{tabular}{|lccccc|}
\hline \multirow{2}{*}{ Competencias profesionales } & \multicolumn{2}{c}{ Desarrollo } & \multicolumn{2}{c}{ Relevancia } & \multirow{2}{*}{ Sig (p) } \\
& Media & Desv. típ. & Media & Desv. típ. & Wilcoxon \\
\hline Conciencia de uno mismo (C1) & 3.67 & 0.973 & 4.26 & 0.865 & $<.001$ \\
\hline Análisis, síntesis y crítica (C2) & 3.43 & 0.905 & 4.19 & 0.906 & $<.001$ \\
\hline Organización y planificación (C3) & 3.72 & 0.964 & 4.42 & 0.716 & $<.001$ \\
\hline Habilidades de comunicación (C4) & 3.75 & 1.005 & 4.55 & 0.678 & $<.001$ \\
\hline Responsabilidad y perseverancia (C5) & 3.79 & 1.049 & 4.37 & 0.876 & $<.001$ \\
\hline Toma de decisiones (C6) & 3.52 & 0.997 & 4.27 & 0.811 & $<.001$ \\
\hline Orientación a las personas (C7) & 3.33 & 1.075 & 4.24 & 0.879 & $<.001$ \\
\hline Trabajo en equipo y cooperación (C8) & 3.99 & 0.961 & 4.39 & 0.890 & $<.001$ \\
\hline Capacidad de aprendizaje y adaptación (C9) & 3.64 & 1.016 & 4.49 & 0.693 & $<.001$ \\
\hline Flexibilidad y orientación al cambio (C10) & 3.58 & 1.042 & 4.27 & 0.846 & $<.001$ \\
\hline Motivación por el logro (C11) & 3.37 & 1.072 & 4.20 & 0.920 & $<.001$ \\
\hline Compromiso con la organización (C12) & 3.52 & 1.007 & 4.02 & 0.884 & $<.001$ \\
\hline Capacidad para trabajar bajo presión (C13) & 3.62 & 0.980 & 4.04 & 0.985 & $<.001$ \\
\hline $\begin{array}{l}\text { Resolución de conflictos y técnicas de } \\
\text { negociación (C14) }\end{array}$ & 3.38 & 1.046 & 4.34 & 0.850 & $<.001$ \\
\hline Búsqueda de la excelencia (C15) & 3.43 & 1.087 & 4.09 & 0.921 & $<.001$ \\
\hline Innovación (C16) & 3.21 & 1.058 & 4.38 & 0.774 & $<.001$ \\
\hline Emprendimiento (C17) & 3.15 & 1.178 & 4.08 & 1.006 & $<.001$ \\
\hline Liderazgo (C18) & 3.22 & 1.042 & 3.89 & 1.017 & $<.001$ \\
\hline $\begin{array}{l}\text { Capacidad de resiliencia y tolerancia a la } \\
\text { frustración (C19) }\end{array}$ & 3.18 & 1.209 & 4.20 & 0.899 & $<.001$ \\
\hline
\end{tabular}

En cuanto al segundo objetivo: identificar y contrastar posibles diferencias significativas en el desarrollo y relevancia de estas competencias en función de la universidad en la que se cursa el Grado en Educación Primaria, se constata que existen diferencias (ver Figura 1)

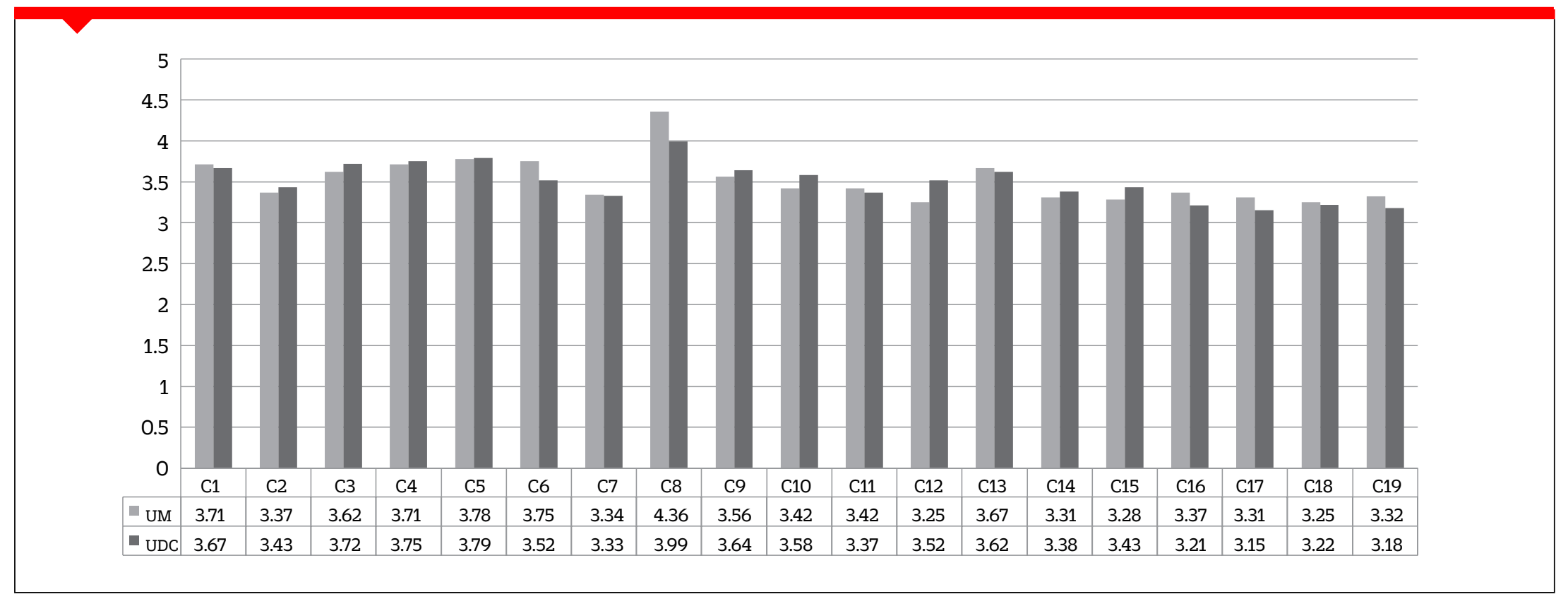

Figura 1. Desarrollo de las competencias profesionales en la UM y UDC 
Para determinar si estas diferencias del nivel de desarrollo de las competencias profesionales en la UM y en la UDC son estadísticamente significativas se realiza un análisis en profundidad, a través de la prueba U Mann-Withney para dos muestras independientes, que determina que solo existen diferencias estadísticamente significativas ( $p \leq .05)$, en cuanto al nivel de desarrollo en la competencia "trabajo en equipo y la cooperación" ( $Z=-3.048, p=.002)$ (ver Tabla 5).

\section{Tabla 5}

Desarrollo de las competencias profesionales en la UM y la UDC

\begin{tabular}{|c|c|c|c|c|}
\hline Competencias profesionales & Universidad & $\begin{array}{c}\text { Rango } \\
\text { promedio }\end{array}$ & $\begin{array}{l}\text { Suma de } \\
\text { rangos }\end{array}$ & $\begin{array}{c}\text { Z Sig. } \\
\text { asintót. } \\
\text { (bilateral) }\end{array}$ \\
\hline \multirow[t]{2}{*}{ Conciencia de uno mismo } & Universidad de Murcia & 106.97 & 11018.00 & -.490 \\
\hline & Universidade da Coruña & 103.08 & 10927.00 & .624 \\
\hline \multirow[t]{2}{*}{ Análisis, síntesis y crítica } & Universidad de Murcia & 103.71 & 10785.50 & -.449 \\
\hline & Universidade da Coruña & 107.26 & 11369.50 & .653 \\
\hline \multirow[t]{2}{*}{ Organización y planificación } & Universidad de Murcia & 102.85 & 10696.00 & -.660 \\
\hline & Universidade da Coruña & 108.10 & 11459.00 & .510 \\
\hline \multirow[t]{2}{*}{ Habilidades de comunicación } & Universidad de Murcia & 103.76 & 10791.50 & -.430 \\
\hline & Universidade da Coruña & 107.20 & 11363.50 & .667 \\
\hline \multirow[t]{2}{*}{ Responsabilidad y perseverancia } & Universidad de Murcia & 104.36 & 10853.00 & -.283 \\
\hline & Universidade da Coruña & 106.62 & 11302.00 & .777 \\
\hline \multirow[t]{2}{*}{ Toma de decisiones } & Universidad de Murcia & 112.11 & 11659.50 & -1.631 \\
\hline & Universidade da Coruña & 99.01 & 10495.50 & .103 \\
\hline \multirow[t]{2}{*}{ Orientación a las personas } & Universidad de Murcia & 105.94 & 11017.50 & -.107 \\
\hline & Universidade da Coruña & 105.07 & 11137.50 & .914 \\
\hline \multirow[t]{2}{*}{ Trabajo en equipo y cooperación } & Universidad de Murcia & 117.50 & 12219.50 & -3.048 \\
\hline & Universidade da Coruña & 93.73 & 9935.50 & .002 \\
\hline \multirow[t]{2}{*}{ Capacidad de aprendizaje y adaptación } & Universidad de Murcia & 103.08 & 10720.50 & -.594 \\
\hline & Universidade da Coruña & 107.87 & 11434.50 & .552 \\
\hline \multirow[t]{2}{*}{ Flexibilidad y orientación al cambio } & Universidad de Murcia & 99.99 & 10398.50 & -1.365 \\
\hline & Universidade da Coruña & 110.91 & 11756.50 & .172 \\
\hline \multirow[t]{2}{*}{ Motivación por el logro } & Universidad de Murcia & 107.08 & 11136.00 & -.387 \\
\hline & Universidade da Coruña & 103.95 & 11019.00 & .699 \\
\hline \multirow[t]{2}{*}{ Compromiso con la organización } & Universidad de Murcia & 98.63 & 10257.00 & -1.697 \\
\hline & Universidade da Coruña & 112.25 & 11898.00 & .090 \\
\hline \multirow[t]{2}{*}{ Capacidad para trabajar bajo presión } & Universidad de Murcia & 109.20 & 11356.50 & -.906 \\
\hline & Universidade da Coruña & 101.87 & 10798.50 & .365 \\
\hline \multirow{2}{*}{$\begin{array}{l}\text { Resolución de conflictos y técnicas de } \\
\text { negociación }\end{array}$} & Universidad de Murcia & 104.20 & 10836.50 & -.321 \\
\hline & Universidade da Coruña & 106.78 & 11318.50 & .748 \\
\hline \multirow[t]{2}{*}{ Búsqueda de la excelencia } & Universidad de Murcia & 102.06 & 10614.50 & -.843 \\
\hline & Universidade da Coruña & 108.87 & 11540.50 & .399 \\
\hline \multirow[t]{2}{*}{ Innovación } & Universidad de Murcia & 110.79 & 11522.50 & -1.299 \\
\hline & Universidade da Coruña & 100.31 & 10632.50 & .194 \\
\hline
\end{tabular}




\begin{tabular}{|llccc|}
\hline \multirow{2}{*}{ Competencias profesionales } & \multicolumn{1}{c}{ Universidad } & $\begin{array}{c}\text { Rango } \\
\text { promedio }\end{array}$ & $\begin{array}{c}\text { Suma de } \\
\text { rangos }\end{array}$ & $\begin{array}{c}\text { Z Sig. } \\
\text { asintót. } \\
\text { (bilateral) }\end{array}$ \\
\hline \multirow{2}{*}{ Emprendimiento } & Universidad de Murcia & 109.19 & 11356.00 & -.902 \\
\cline { 2 - 4 } & Universidade da Coruña & 101.88 & 10799.00 & .367 \\
\hline Liderazgo & Universidad de Murcia & 107.60 & 11190.00 & -.514 \\
\cline { 2 - 4 } & Universidade da Coruña & 103.44 & 10965.00 & .607 \\
\hline $\begin{array}{l}\text { Capacidad de resiliencia y tolerancia } \\
\text { a la frustración }\end{array}$ & Universidad de Murcia & 109.52 & 11390.50 & -.980 \\
\cline { 2 - 4 } & Universidade da Coruña & 101.55 & 10764.50 & .327 \\
\hline
\end{tabular}

De igual modo, se afirma que existen diferencias entre la percepción que tiene el alumnado de la Universidad de Murcia y la Universidade da Coruña en cuanto a la relevancia conferida a las competencias profesionales para su futuro proceso de inserción laboral (ver Figura 2).

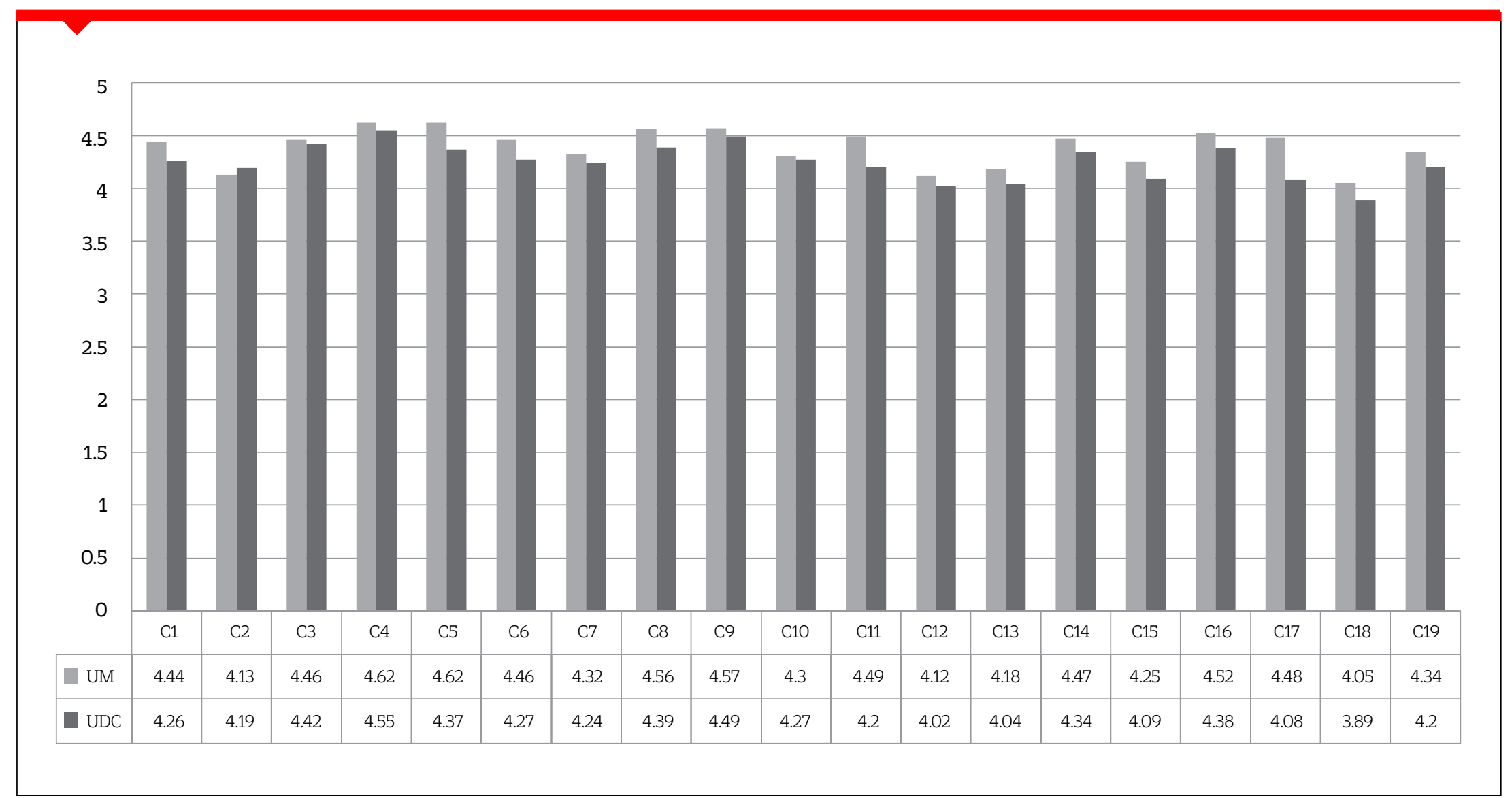

Figura 2. Relevancia de las competencias profesionales en la UM y UDC

Sin embargo, aunque estas diferencias son observables, se puede determinar que en la mayor parte de ellas existe acuerdo entre los estudiantes al considerarlas importantes para la inserción en el mercado laboral, ya que la prueba U de Mann-Whitney para dos muestras independientes, no ofrece diferencias estadísticamente significativas para 16 de las 19 competencias analizadas. Esta significatividad ( $p \leq .05)$, se encuentra en relación con la "responsabilidad y perseverancia" ( $p=.043$ ), "motivación por el logro" ( $p=.019) \mathrm{y}$ "emprendimiento" ( $p=.003$ ), competencias que el alumnado de la Universidade Murcia le da mayor relevancia (ver Tabla 6). 
Tabla 6

Relevancia de las competencias profesionales en la UM y la UDC

\begin{tabular}{|c|c|c|c|c|}
\hline Competencias profesionales & Universidad & $\begin{array}{c}\text { Rango } \\
\text { promedio }\end{array}$ & $\begin{array}{l}\text { Suma de } \\
\text { rangos }\end{array}$ & $\begin{array}{c}\text { ZSig. } \\
\text { asintót. } \\
\text { (bilateral) }\end{array}$ \\
\hline \multirow{2}{*}{ Conciencia de uno mismo } & Universidad de Murcia & 111.41 & 11587.00 & -1.548 \\
\hline & Universidade da Coruña & 99.70 & 10568.00 & .122 \\
\hline \multirow{2}{*}{ Análisis, síntesis y crítica } & Universidad de Murcia & 101.88 & 10596.00 & -.916 \\
\hline & Universidade da Coruña & 109.05 & 11559.00 & .360 \\
\hline \multirow{2}{*}{ Organización y planificación } & Universidad de Murcia & 106.23 & 11048.00 & -.194 \\
\hline & Universidade da Coruña & 104.78 & 11107.00 & .846 \\
\hline \multirow{2}{*}{ Habilidades de comunicación } & Universidad de Murcia & 109.18 & 11354.50 & -1.057 \\
\hline & Universidade da Coruña & 101.89 & 10800.50 & .290 \\
\hline \multirow{2}{*}{ Responsabilidad y perseverancia } & Universidad de Murcia & 112.87 & 11738.50 & -2.021 \\
\hline & Universidade da Coruña & 98.27 & 10416.50 & .043 \\
\hline \multirow{2}{*}{ Toma de decisiones } & Universidad de Murcia & 111.81 & 11628.50 & -1.655 \\
\hline & Universidade da Coruña & 99.31 & 10526.50 & .98 \\
\hline \multirow{2}{*}{ Orientación a las personas } & Universidad de Murcia & 107.76 & 11207.00 & -.582 \\
\hline & Universidade da Coruña & 103.28 & 10948.00 & .561 \\
\hline \multirow{2}{*}{ Trabajo en equipo y cooperación } & Universidad de Murcia & 110.81 & 11524.50 & -1.457 \\
\hline & Universidade da Coruña & 100.29 & 10630.50 & .145 \\
\hline \multirow{2}{*}{ Capacidad de aprendizaje y adaptación } & Universidad de Murcia & 109.10 & 11346.00 & -.998 \\
\hline & Universidade da Coruña & 101.97 & 10809.00 & .318 \\
\hline \multirow{2}{*}{ Flexibilidad y orientación al cambio } & Universidad de Murcia & 106.10 & 11034.00 & -.154 \\
\hline & Universidade da Coruña & 104.92 & 11121.00 & .878 \\
\hline \multirow{2}{*}{ Motivación por el logro } & Universidad de Murcia & 114.45 & 11902.50 & -2.342 \\
\hline & Universidade da Coruña & 96.72 & 10252.50 & .019 \\
\hline \multirow{2}{*}{ Compromiso con la organización } & Universidad de Murcia & 109.13 & 11349.50 & -.920 \\
\hline & Universidade da Coruña & 101.94 & 10805.50 & .357 \\
\hline \multirow{2}{*}{ Capacidad para trabajar bajo presión } & Universidad de Murcia & 109.81 & 11420.50 & -1.086 \\
\hline & Universidade da Coruña & 101.27 & 10734.50 & .277 \\
\hline \multirow{2}{*}{$\begin{array}{l}\text { Resolución de conflictos y técnicas de } \\
\text { negociación }\end{array}$} & Universidad de Murcia & 109.93 & 11432.50 & -1.179 \\
\hline & Universidade da Coruña & 101.16 & 10722.50 & .239 \\
\hline \multirow{2}{*}{ Búsqueda de la excelencia } & Universidad de Murcia & 109.75 & 11414.00 & -1.075 \\
\hline & Universidade da Coruña & 101.33 & 10741.00 & .282 \\
\hline \multirow{2}{*}{ Innovación } & Universidad de Murcia & 109.55 & 11393.00 & -1.080 \\
\hline & Universidade da Coruña & 101.53 & 1076200 & .280 \\
\hline \multirow{2}{*}{ Emprendimiento } & Universidad de Murcia & 116.92 & 12160.00 & -2.958 \\
\hline & Universidade da Coruña & 94.29 & 9995.00 & .003 \\
\hline \multirow{2}{*}{ Liderazgo } & Universidad de Murcia & 110.19 & 11459.50 & -1.165 \\
\hline & Universidade da Coruña & 100.90 & 10695.50 & .244 \\
\hline \multirow{2}{*}{$\begin{array}{l}\text { Capacidad de resiliencia y tolerancia } \\
\text { a la frustración }\end{array}$} & Universidad de Murcia & 109.88 & 11428.00 & -1.128 \\
\hline & Universidade da Coruña & 101.20 & 10727.00 & .259 \\
\hline
\end{tabular}


Por último, se persigue como tercer objetivo: considerar y evaluar la percepción que manifiesta el alumnado respecto de su preparación para enfrentarse al proceso de inserción socio-laboral en ambas universidades. Para ello, se tienen en cuenta las respuestas efectuadas por el alumnado ante la pregunta ite sientes preparado para enfrentar tu próxima inserción laboral? Los resultados permiten afirmar que, tanto en la muestra encuestada en la UM como en la UDC, expresa cierto grado de incertidumbre ante esa futura, y cada vez más cercana, inserción laboral que hace que alrededor del 40\% del alumnado de ambas instituciones no se sienta certeramente preparado. Si bien, cabe decir que los valores positivos de la escala (bastante y mucho) aglutinan el 54.81\% de las respuestas en el caso de la UM y el $43.7 \%$ en la UDC.

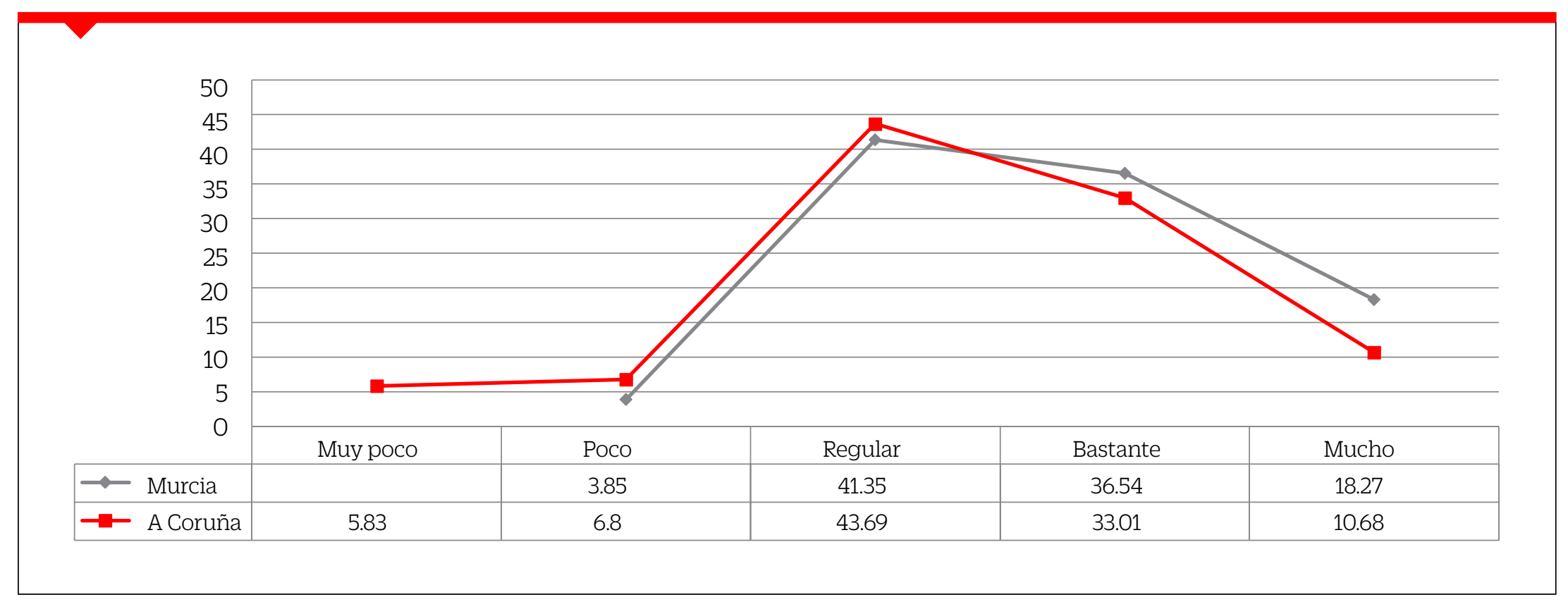

Figura 3. ¿Te sientes preparado para enfrentar tu próxima inserción laboral?

Como muestra la Figura 3, se observan diferencias entre las valoraciones efectuadas por el alumnado de la Universidad de Murcia y el de la Universidade da Coruña, siendo estos últimos los que tienen una percepción más negativa, ya que el 12.63\% de ellos se sienten muy poco o poco preparados. Estas diferencias se corroboran al aplicar la prueba U de Mann-Whitney que evidencia que los estudiantes de la UM tiene mayores expectativas frente a su incursión en el mercado laboral $(p=.024)$.

\section{DISCUSIÓN}

A través de los resultados obtenidos y del análisis de los datos aportados por una muestra de alumnado de último curso de la Universidad de Murcia y la Universidade da Coruña de la titulación de Educación Primaria, se desprende una serie de conclusiones a raíz de los objetivos que se perseguían. 
Los estudiantes consideran, y coinciden en ello en ambos centros, que a lo largo de los cuatro años han desarrollado en gran medida las competencias profesionales para su proceso de inserción socio-laboral, siendo el "trabajo en equipo y cooperación” el más trabajado, junto con la "toma de decisiones" y las "habilidades de comunicación" y con menos énfasis el "liderazgo" y el "compromiso con la organización". Así mismo, la muestra le ha asignado el mayor grado de importancia al "trabajo en equipo y cooperación” y en menor grado, al "liderazgo". Los resultados obtenidos, tanto a nivel de desarrollo competencial a lo largo de la titulación como de relevancia, son coincidentes con el estudio realizado por Alonso-Martín et al. (2011), con alumnado de la Universidad Complutense de Madrid y la Universidad de Huelva. Del mismo modo, la investigación desarrollada por Lluch Molins, Fernández-Ferrer, Pons Seguí y Cano García (2017), con una muestra de egresados de las universidades de Barcelona, Politécnica de Cataluña, Rovira i Virgili, Autónoma de Barcelona, Islas Baleares, Granada y Oberta de Cataluña, demostró que las competencias que consideraban más importantes para su carrera profesional y a la vez han sido desarrolladas en mayor medida eran el "trabajo en equipo" y la "capacidad comunicativa".

El alumnado de la UM y de la UDC percibe un nivel de desarrollo de competencias profesionales para la empleabilidad menor que la relevancia que le ha otorgado, resultados en la línea de la investigación de Muñoz Cantero, Rebollo Quintela y Espiñeira Bellón (2014) con discentes de UDC de $4^{\circ}$ de Educación Primaria en el curso 2012/2013 y el estudio de AlonsoMartín et. al (2011). Así mismo, Lluch Molins et al. (2017) destaca que "los egresados no toman conciencia de haber desarrollado determinadas competencias hasta que entran en contacto con el mundo laboral" (p. 57), razón que motiva que el alumnado otorgue un mayor nivel de relevancia que de desarrollo. Esta idea viene a apoyar lo expuesto por diversos autores (Prats, 2016; Rodríguez-Gómez et al., 2017; Rosales, 2013), que coinciden en afirmar que la adquisición de las competencias no se realiza únicamente en la formación inicial, sino que requiere del acceso a la función docente y del proyecto de carrera profesional.

Teniendo en cuenta que el Grado en Educación Primaria es un título que conduce a una profesión regulada y que las universidades diseñan su plan de estudios de acuerdo con los requerimientos de una orden ECI Ministerial, en concreto la Orden ECI /3857/2007, de 27 de diciembre, por la que se establecen los requisitos para la verificación de los títulos universitarios oficiales que habiliten para el ejercicio de la profesión de Maestro en Educación Primaria, se determinó en qué medida difiere el desarrollo de las competencias que demanda el mercado laboral en función de la institución de educación superior de pertenencia. Se constata que realmente existen diferencias entre la Universidad de Murcia y la Universidade da Coruña, pero aplicada la prueba U de Mann-Whitney evidenciamos que solo son estadísticamente significativas en la competencia trabajo en equipo y cooperación en su nivel de desarrollo, siendo superior en la Universidad de Murcia. Esta falta de significatividad, viene a constatar que el nivel de desarrollo competencial entre el alumnado que cursa sus estudios en diferentes 
centros debe ser igual cuando se trata de una profesión regulada por el estado español, como es la de maestro en Educación Primaria.

En relación con la relevancia otorgada por el alumnado de ambas instituciones, existen diferencias en todas las competencias y son estadísticamente significativas para la "responsabilidad y perseverancia", la "motivación por el logro" y el "emprendimiento". Esta diferencia entre el alumnado de la UDC y la UM puede ser debida a que aunque las percepciones sobre rol docente son forjadas por el alumnado a título individual, estas pueden estar influenciadas por las concepciones transmitidas desde las universidades, y condicionadas también por las características inherentes al mercado laboral de cada Comunidad Autónoma. Para poder determinar qué aspectos intervienen, sería conveniente ampliar la investigación con grupos focales en ambas instituciones.

Por último, los datos extraídos del presente trabajo muestran que el futuro maestro no se siente ciertamente preparado para el ejercicio profesional, aspecto coincidente en ambas universidades, pero más notorio y estadísticamente significativo es el caso de la Universidade da Coruña, en la que solo el $43.7 \%$ de la muestra se considera bastante o muy preparado para afrontar su incursión en el mercado laboral. Estos resultados están en consonancia con lo expuesto por Herraiz (2015) y Bozu y Aránega Español (2017) en el que los discentes perciben no tener "el nivel de preparación que se requeriría para dar respuestas a los problemas actuales y desafiantes de la sociedad y de la profesión docente" (p.158), a lo que suman la urgente necesidad de una modificación en los planes de estudios y con ellos en las competencias profesionales de los mismos, para hacer frente a la sociedad actual.

Una sociedad caracterizada por la globalidad, la complejidad, la incertidumbre y en constante cambio, que plasma todas sus particularidades en el mercado laboral, no puede perseguir que las instituciones de educación superior formen profesionales con perfiles laborales prefijados de antemano y con unas funciones definidas e inmutables en el tiempo y que con ello, se dé una perfecta adecuación entre puestos y trabajadores.

Las universidades, y más en concreto las Facultades de Ciencias de la Educación encargadas de la formación inicial, tienen que capacitar al maestro que será "concebido como un profesional de la enseñanza que se mueve en situaciones complejas, cambiantes, inciertas y conflictivas, que le convierten en un investigador en el aula" (Baelo \& Arias, 2011, p.116). Su misión no ha de ser otra que, actuar de modo proactivo, y que así la formación inicial tienda a desarrollar competencias que les posibiliten, no solo acceder al mercado laboral, sino capacitarlos para que puedan seguir adquiriendo las competencias necesarias para su desarrollo profesional y personal en una sociedad certeramente voluble, facultándolos para un aprendizaje a lo largo de la vida.

El EEES asume una formación basada en competencias, es decir, formar en y para la acción, en la toma de decisiones para convertir el saber (competencia técnica) en saber hacer (competencia metodológica) con dosis de saber estar (competencia participativa) y saber ser 
(competencia personal), de forma que la educación superior tenga una vinculación con las demandas del mundo productivo y laboral, y, más concretamente, con la inserción socio-laboral, ya que la competencia es una demostración de los conocimientos, habilidades, conductas y actitudes requeridas para desempeñar una función o rol profesional de forma eficaz.

En el modelo de enseñanza y aprendizaje que se propone en este espacio europeo común, lo importante no es lo que el maestro sabe o hace (concepción tradicional de la enseñanza), sino qué aprenden y cómo lo aprenden los estudiantes (el aprendizaje de competencias).

De este modo, se podrá afirmar y no dejarlo en una mera suposición, lo recogido hace más de una década por el Ministerio de Educación y Ciencia (MEC, 2006) "los títulos deben preparar para el acceso al ejercicio profesional, es decir, deben tener como objetivo la amplia empleabilidad de sus titulados" (p. 7), sin olvidarnos que el maestro en educación primaria, no solo puede tener el centro educativo como horizonte único, aunque suele ser el más frecuente, sino que puede abarcar otros ámbitos profesionales (Bozu \& Aránega Español, 2017) y repensar su acción profesional.

\section{REFERENCIAS}

Alonso- Martín, P., Aguilera-García, J. L., Canalejo-Raya, A., Córdoba-García, F. Cruz-Díaz, R., Gil Hernández, S.,... Torronteras-Santiago, R. (2011). Las competencias en el currículum universitario y sus implicaciones para diseñar el aprendizaje y la formación del profesorado. En J.J. Maquilón Sánchez, M.P. García Sanz \& M.L. Belmonte Almagro (Eds.), Innovación educativa en la enseñanza formal (pp. 755-763). Murcia: Asociación Universitaria de Formación del Profesorado.

Baelo, R. \& Arias, A.R. (2011). La formación de maestros en España, de la teoría a la práctica. Tendencias pedagógicas, 18,105-131. Recuperado de https://goo.gl/C4kv1L

Bozu, Z. \& Aránega Español, S. (2017). La formación inicial de maestros y maestras a debate: ¿qué nos dicen sus protagonistas? Profesorado. Revista de currículum y formación del profesorado, 21 (1), 143-163. Recuperado de https://goo.gl/Xnku6m

Contreras, M. (2014). Formación universitaria e inserción laboral. El caso de los graduados por la Escuela Universitaria de Magisterio “Sagrada Familia” de Úbeda (Jaén). Revista Electrónica de Investigación y Docencia, 8, 73-92. Recuperado de https://goo.gl/8LxgKZ

Grover, V. K. (2015). Identification of best practices in transfer of training in teacher education as perceived by teacher educators. International Journal of Applied Research, 1(7), 204-209. Recuperado de https://goo.gl/8gowhC

Helyer, R., \& Lee, D. (2014). The role of work experience in the future employability of higher education graduates. Higher Education Quarterly, 68(3), 348-372. doi: http://dx.doi.org/10.1111/hequ.12055

Hernández, R., Fernández, C., \& Baptista, P. (2007). Metodología de investigación (4a ed.). México: McGraw-Hill.

Herraiz, F. (2015). Entre la Formación Inicial en la Universidad y la vida laboral en la Escuela de maestros y maestras noveles. Reflexionando en torno a identidades docentes y sus aprendizajes. Profesorado. Revista de Currículum y Formación del Profesorado, 19(2), 203-2014. Recuperado de https://goo.gl/mcBBUw 
Hudson, B., Zgaga, P., \& Astrand, B. (Eds.) (2010). Advancing quality cultures for teacher education in Europe: Tensions and opportunities. Sweden: Umea School of Education, University of Umea.

Lluch Molins, L., Fernández-Ferrer, M., Pons Seguí, L. \& Cano García, E. (2017). Competencias profesionales de los egresados universitarios: estudio de casos en cuatro titulaciones. Revista Qurriculum, 30, 49-64. Recuperado de https://riull.ull.es/xmlui/handle/915/6135

Martínez Clares, P., \& González Lorente, C. (2018). Validez de contenido y consistencia interna de un cuestionario sobre el proceso de inserción socio-laboral desde la mirada del universitario. Revista Complutense de Educación, 29(3), 33-50. doi: http://dx.doi.org/10.5209/RCED.53721

MEC (2006). Propuesta Directrices para la elaboración de títulos universitarios de grado y master. Recuperado de http://firgoa.usc.es/drupal/files/directrices.pdf

Medina, J.L., \& Pérez Cabrera, M.J. (2017). La construcción del conocimiento en el proceso de aprender a ser profesor: la visión de los protagonistas. Profesorado. Revista de Currículum y Formación del Profesorado, 21(1), 17-38. Recuperado de https://goo.gl/iD1Pr2

Mendoza, M., \& Covarrubias, C.G. (2014). Competencias profesionales movilizadas en el prácticum: percepciones del estudiantado del grado de maestro en educación primaria. Revista Actualidades Investigativas en Educación, 14(3), 1-24. doi: http://dx.doi.org/10.15517/AIE.V14I3.16089

Muñoz Cantero, J.M, Rebollo-Quintela, N. \& Espiñeira-Bellón, E.M. (2014). Percepción de competencias en el EEES: análisis en el Grado de Educación Primaria. Revista Electrónica Interuniversitaria de Formación del Profesorado, 17 (3), 123-139. doi: http://dx.doi.org/10.6018/reifop.17.3.204091

Pineda, P., Agud, I., \& Ciraso, A. (2016). Factores que intervienen en la inserción laboral de los titulados en Educación en tiempos de crisis. Revista de educación, 372(4-6), 141-168. doi: http://10.4438/1988-592X-RE-2015-372-318

Prats, E. (2016). La formación inicial docente entre profesionalismo y vías alternativas: mirada internacional. Bordón. Revista de Pedagogía, 68 (2), 19-33. doi: https://doi.org/10.13042/Bordon.2016.68202

Rodríguez-Gómez, D., Armengol, C., \& Meneses, J. (2017). La adquisición de las competencias profesionales a través de las prácticas curriculares de la formación inicial de maestros. Revista de Educación, 376, 229-251. doi: http:// dx.doi.org/10.4438/1988-592X-RE-2017-376-350

Rosales, C. (2013). Competencias específicas curriculares que ha de adquirir el estudiante del título de grado de maestro. Profesorado. Revista de currículum y formación del profesorado, 17(3), 73-90. Recuperado de https://goo.gl/ WHwWHv

Stiwne, E., \& Alves, M. (2010). Higher education and employability of graduates: Will Bologna make a difference? European Educational Research Journal, 9(1), 2-44. doi: https://doi.org/10.2304/eerj.2010.9.1.32

RIDU / Revista Digital de Investigación en Docencia Universitaria / ISNN 2223-2516

( ) Los autores. Este artículo es publicado por la Revista Digital de Investigación en Docencia Universitaria del Área de Institutional Research and Effectiveness de la Dirección de Aseguramiento de la Calidad, Universidad Peruana de Ciencias Aplicadas. Este es un artículo de acceso abierto, distribuido bajo los términos de la LicenciaCreativeCommons Atribución-CompartirIgual 4.0 Internacional. ( http://creativecommons.org/licenses/by-sa/4.0/), que permite el uso no comercial, distribución y reproducción en cualquier medio, siempre que la obra original sea debidamente citada. 